\title{
Joint And Muscle Forces During Clenching
}

\author{
Stefan Rues*1, Jürgen Lenz ${ }^{1}$, Hans J. Schindler ${ }^{1}$, and Karl Schweizerhof ${ }^{2}$ \\ ${ }^{1}$ Research Group Biomechanics, University of Karlsruhe (TH), Kaiserstraße 12, 76128 Karlsruhe \\ ${ }^{2}$ Institute for Mechanics, University of Karlsruhe (TH), Kaiserstraße 12, 76128 Karlsruhe
}

\begin{abstract}
The masticatory system is highly redundant. Therefore, complete knowledge about the activation patterns of the chewing muscles belonging to a specific resultant bite force can only be gained either by simultaneous force- and EMG-measurement or with the help of optimization strategies. In this study, such EMG and force measurements were carried out with 10 test persons and the results compared to those computed with several objective functions.

The results show an increase of the joint forces with an increase of the horizontal component of the resultant bite force. The test persons seem to favor energy minimization as control mechanism.
\end{abstract}

() 2006 WILEY-VCH Verlag GmbH \& Co. KGaA, Weinheim

\section{Introduction}

The human masticatory system consists of twelve essential muscles connecting the mandible to the maxilla. Each muscle can generate a force vector with an a priori unknown magnitude but a line of action which can approximately be constructed from its geometry. For the joint forces, however, the magnitude as well as the line of action are unknown. Thus, there are 12 (muscle forces) +6 (joint force components) $=18$ unknown parameters in comparison with 6 equilibrium conditions. Therefore, the system is highly redundant, i.e., a specific resultant force can be generated by an infinite variety of activation patterns. In this study, the activation patterns and the resultant bite force were measured simultaneously during specific clenching tasks. The results based on the measured data were compared to those calculated with the help of different optimization strategies.

\section{Materials and Methods}

So far, no data were available from experiments in which the activities of all masticatory muscles and the resultant force had been recorded simultaneously. This complete knowledge is, however, indispensable to determine the direction and amount of the reaction forces transferred to the condyles.

For that purpose, in ten healthy male subjects (average age: $29 \pm 2.6$ years) the intraoral force transfer and the electromyographic activities of the masseter, anterior and posterior temporalis, medial and lateral pterygoid, and anterior digastric were recorded simultaneously in simulated clenching tasks during the generation of various resulting force vectors [1]. A feedback system enabled the test persons to perform 19 specific clenching tasks (circumferential angle $\varphi=0^{\circ}, 60^{\circ}, 90^{\circ}, 180^{\circ}, 270^{\circ}$, $300^{\circ}$; cranial angle $\theta=0^{\circ}, 20^{\circ}, 40^{\circ}, 60^{\circ}$ with respect to the normal z' on the occlusal plane) at different magnitudes of the resultant force $\mathrm{F}_{\text {res }}$ (cf. Fig. 1, left). The centrally transmitted resultant force was determined with an intraoral measuring device also illustrated in Fig. 1 (bearing pin device fixed on custom made metal splints and equipped with strain gauges).
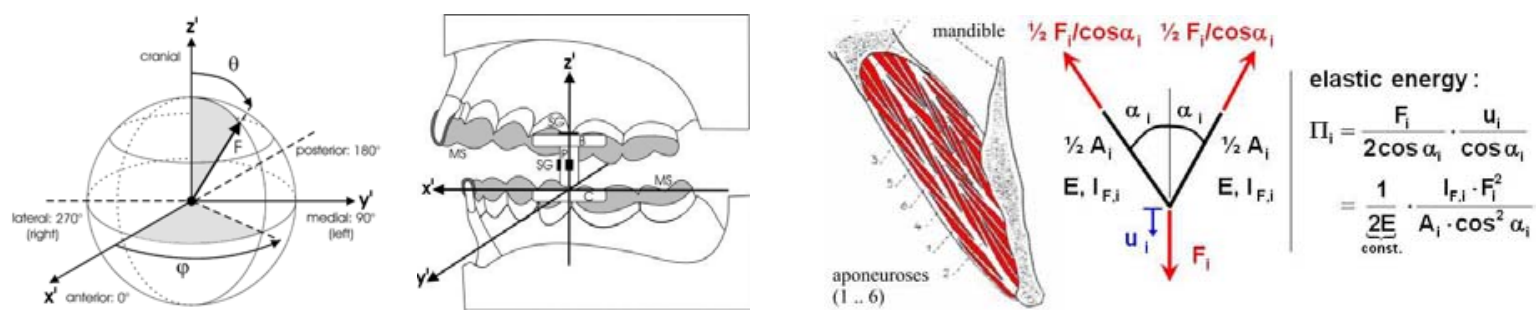

Fig. 1 Left: Used coordinate systems and force measuring device. Right: Determination of a muscle's elastic energy.

Additionally, for each test person a 3D-model of the musculature was constructed using horizontal and frontal magnetic resonance tomograms (MRTs) which also served to identify the occlusal plane as well as the position of the bearing pin. From these models the centroids of the muscles' origin and insertion areas whose connection lines coincide with the muscles' lines of action for a homogeneous activation, were determined as well as the physiological cross-sectional areas $A_{\mathrm{i}}=V_{\mathrm{i}} / l_{\mathrm{f}, \mathrm{i}}\left(V_{\mathrm{i}}\right.$ : volume of the contractile tissue of the $\mathrm{i}^{\text {th }}$ muscle, $\mathrm{l}_{\mathrm{f}, \mathrm{i}}$ : fibre length, values taken from [2]). Now, a force law relating the muscle forces to the EMG-activities [3] can be derived and all remaining unknowns be computed under the assumptions that the mandible behaves like a rigid body and that joint forces can only be transferred to the fossa mandibularis by compression.

\footnotetext{
* Corresponding author: e-mail: stefan.rues@ifm.uni-karlsruhe.de, Phone: +49 721608 2615, Fax: +49 7216087990
} 
All objective functions but one were taken from literature (e.g. [4], [5]). In this study, additionally the elastic energy of the contractile tissue of a pennated muscle (pennation angle $\alpha_{\mathrm{i}}$ ) was derived as elucidated in Fig. 1 (right). Most authors, namely, only take into account the squared muscle force as an equivalent for the elastic energy.

\section{Results}

Over all tasks and all test persons, the intrinsic muscle stress was found to be $\mathrm{P}=0.32 \pm 0.13 \mathrm{~N} / \mathrm{mm}^{2}$. This corresponds well with the value $\mathrm{P}=0.37 \mathrm{~N} / \mathrm{mm}^{2}$ given in [6]. The results displayed in Fig. 2 show that the interval of joint force magnitudes ranges from about 60 to $100 \%$ of the resultant bite force. Highest joint forces occur under large angles $\theta$, i.e., under bite forces with marked horizontal components. Furthermore, muscles whose lines of action nearly coincide with the actual bite force direction are highly activated. This behavior can best be observed for the lateral pterygoid muscle.
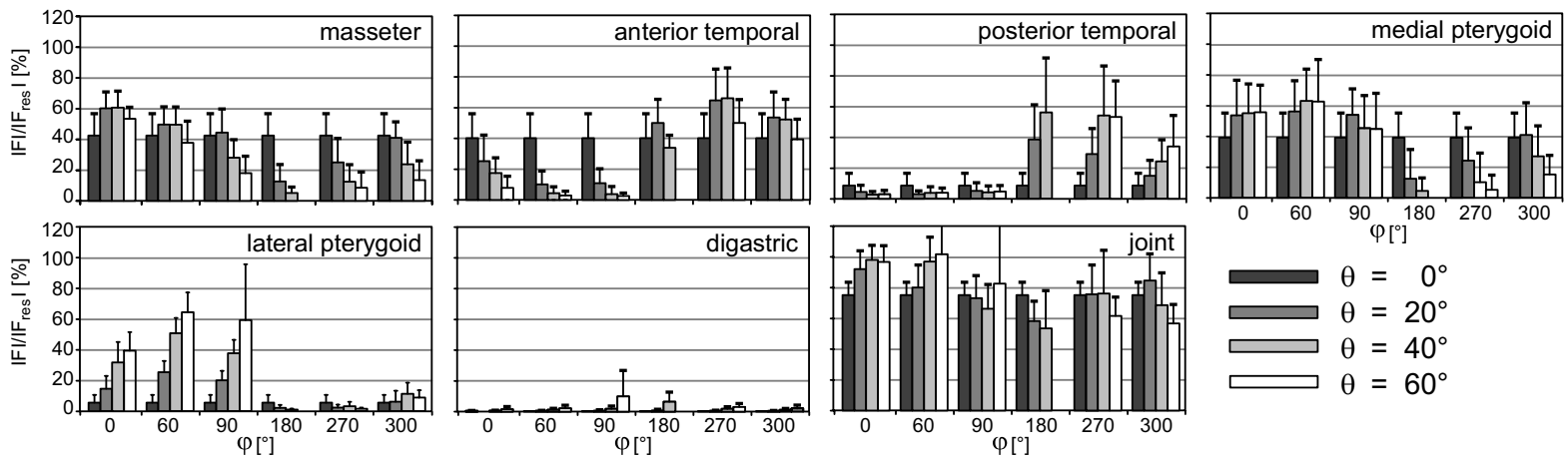

Fig. 2 Mean muscle and joint forces normalized to the resultant bite force $\left(\mathrm{F}_{\mathrm{res}}=150 \mathrm{~N}\right)$ for the right side of all test persons.

Table 1 summarizes the mean differences $\Delta \mathrm{F}_{\text {mean }}$ between the calculations based on the measured data (as reference) and the results gained with the help of different optimization strategies. Best correlation is found for the proposed minimization of the elastic energy of the contractile tissue.

Table 1 Differences between results based on optimization strategies and those based on the measured data $\left(\mathrm{F}_{\text {res }}=150 \mathrm{~N}\right)$

\begin{tabular}{|c|c|c|c|c|c|c|c|}
\hline opt. strategy & joint forces & muscle forces & $\max$. activity & - & - & 'el. energy' & el. energy \\
\hline obj. function & $\Sigma \mathrm{J}_{\mathrm{i}}$ & $\Sigma \mathrm{F}_{\mathrm{i}}$ & $\max \left(\mathrm{F}_{\mathrm{i}}\right)$ & $\sum \frac{\mathrm{F}_{\mathrm{i}}^{2}}{\mathrm{~A}_{\mathrm{i}}^{2}}$ & $\Sigma \frac{\mathrm{F}_{\mathrm{i}}^{3}}{\mathrm{~A}_{\mathrm{i}}^{3}}$ & $\Sigma \mathrm{F}_{\mathrm{i}}^{2}$ & $\Sigma \frac{\mathrm{F}_{\mathrm{i}}^{2} \cdot l_{\mathrm{F}, \mathrm{i}}}{\mathrm{A}_{\mathrm{i}} \cdot \cos ^{2} \alpha_{\mathrm{i}}}$ \\
\hline$\Delta \mathrm{F}_{\text {mean }}[N]$ & 27.4 & 23.8 & 18.7 & 17.4 & 16.6 & 16.2 & 14.7 \\
\hline
\end{tabular}

\section{Conclusions}

For the intrisic muscle stress a mean value $\mathrm{P}=0.32 \mathrm{~N} / \mathrm{mm}^{2}$ was determined. High joint forces occurred under large angles $\theta$ with magnitudes partially greater than that of the resultant bite force. The test persons favored energy minimization as control mechanism.

Acknowledgements The authors express their gratitude to cand. ing. Frank Eckardt and cand. math. Andrea Otzen for their assistance.

\section{References}

[1] H. J. Schindler, S. Rues, J. C. Türp, J. Lenz, Activity patterns of the masticatory muscles during feedback-controlled simulated clenching activities, Eur. J. Oral Sci. 113, 469-478 (2005).

[2] T. M. G. J. van Eijden, J. A. M. Korfage, P. Brugman, Architecture of the human jaw-closing and jaw-opening muscles, The Anatomical Record 248, 464-474 (1997).

[3] S. Rues, H. J. Schindler, J.C. Türp, K. Schweizerhof, J. Lenz, Masticatory muscle and joint forces during clenching based on simultaneous force and EMG measurement, in: 3rd European Medical and Biological Engineering Conference (EMBEC' 05), Proceedings of the International Federation for Medical and Biological Engineering, 11 (2005).

[4] P. G. Trainor, K. R. McLachlan, W. D. McCall, Modelling of forces in the human masticatory system with optimization of the angulations of the joint loads. J. Biomech. 28, 829-43 (1995).

[5] L. R. Iwasaki, P. E. Petsche, W. D. McCall Jr., D. Marx, J. C. Nickel, Neuromuscular objectives of the human masticatory apparatus during static biting. Arch. Oral Biol. 48, 767-77 (2003).

[6] W. A. Weijs, B. Hillen, Cross-sectional areas and estimated intrinsic strength of the human jaw muscles, Acta morph. nederl.-scand., 23, 267-274 (1985). 